institution; but opportunities may also be provided towards the end of the tenure of the fellowship for work on problems in industrial or other laboratories in applied research related to the fundamental research they have previously carried out. For the United Kingdom about twenty-five fellowships will be available, and the selection will be made by the Royal Society. No age-limits are specified, but it is expected that most of the fellowships will be awarded to persons about 26-32 years of age. Candidates who propose to do research in the natural sciences and in the engineering, agricultural and medical sciences, but excluding clinical medicine, will be considered; they must have a doctorate in science or the equivalent experience. Applications, which may be made at any time, will be considered at regular intervals; for the first two sets of applications the closing dates are, respectively, October 1 and November 30 . Application forms and further information can be obtained from the Assistant Secretary, Royal Society, Burlington House, London, W.1.

IN recognition of the contribution to AngloAmerican understanding made by King George VI, the English-Speaking Union of the United States has established memorial fellowships to enable British men and women (aged 17-29 years) to continue their scientific and technical education in the United States. Twenty-five fellowships have been established for the academic year 1954-55, and each is valued at 2,500 dollars. Further information can be obtained from Mrs. D. R. Dalton, King George VI Memorial Fellowships Committee, English-Speaking Union, 37, Charles Street, London, W.1.

\section{Mammoth Hunters in Austria}

THE sixth volume of the Mitteilungen der Prähistorischen Kommission der Österreichischen Akademie der Wissenschaften, published in Vienna, 1952-1953, includes a long article on a group of prehistoric home sites containing a quantity of mammoth bones and stone implements which were investigated some little while ago by the late Drs. J. Bayer and $\mathrm{H}$. Obermaier. The sites are near Langmannersdorf on the Perschling in the region of St. Polten. The implements recall those found at a somewhat similar mammoth-hunter site at Unter Wisternitz in Moravia, some miles from Brno. The fauna comprises bones of wolf, fox, mammoth and some small rodents, as well as those of hare and rhinoceros. The stone industries are of the late Aurignacian (late Gravettian) type normal for the region. They are well illustrated in fifteen full-page drawings. There are also some half-tone illustrations of the sections eut and some of the finds of bones. A detailed inventory of the objects unearthed concludes the paper.

\section{Mutual Inhibitory Effects in Nodule Formation}

P. S. NuTMaN has shown that the number of root nodules formed on clover, in test-tube culture on agar, depends directly upon the volume of the medium and inversely upon the number of seedlings present per unit volume of medium (Ann. Bot., N.S., 17, 65, $95 ; 1953)$. Plants which form nodules early in seedling development inhibit nodule formation on an associated plant more strongly than plants which normally form nodules later. Among all the types of plants examined, for example sparsely or abundantly nodulating plants, the mutual inhibiting effects when grown in pairs were found to be the same, notwithstanding large differences in plant size. Similar inhibitory effects were observed in the presence of non-leguminous plants; for example, lettuce and flax reduced nodulation on clover plants but to a lesser extent than companion clover plants. Comparable results were obtained in experiments with lucerne and vetch. These and other interesting observations are fully described and discussed. The hypothesis is advanced that bacterial infection and nodule formation may be inhibited by specific root secretions.

\section{National Institute of Agricultural Botany, Cam- bridge}

Vox. 6 of the Journal of the National Institute of Agricultural Botany contains, besides various technical papers and results of its cereal and brussels sprouts trials, a full report of the Institute's first erop conference held last December. On this occasion the suitability of different varieties of wheat, fodder-beet and potatoes for growing in Great Britain was discussed, and much information that had been accumulated over a long period of years was brought together for the first time. The subject in each case was considered from a number of aspects, so that the farmer may judge for himself what type of crop may best suit his own set of conditions. The volume also includes the 1950-51 report of the Seed Production Committee and that of the 1952 Conference of Seed Analysts. In an appendix to the latter, a suggestion is made that expression of weed seed impurities by number per ounce rather than percentage by weight, as at present, would give a better indication of the value of a seed sample. Weed seed content also forms the subject of a detailed survey made by the Official Seed Testing Station of the samples submitted to it over a long term of years. The results show that, since 1923, definite changes in the impurities recorded have occurred. These seem to be largely associated with changes in the sources of supply of the crop seed and particularly with the greatly increased home production of grass and clover seed.

\section{Spectrographic Study of the Double-lined Binary System H.D. 27483}

A sPECTROGRAPHIC study of the double-lined binary system H.D. 27483 has been made at the Dominion Astrophysical Observatory, Victoria, B.C., and Ruth $J$. Northcott and K. O. Wright have published the final results from plates taken at the Dominion Astrophysical Observatory, the David Dunlap Observatory and the Mount Wilson Observatory - a total of eighty-eight plates in all-for this system (Contrib. Dom. Astrophys. Obs., Victoria, B.C., No. 25 ; 1952). The orbital elements have been determined, the final period being $3 \cdot 05911$ days and the eccentricity $0.017 \pm 0.006$ (incidentally, it is worth noticing that this is close to the eccentricity of the earth's orbit). The physical characteristics of the system show that the mass of the system is 0.97 that of the sun, the radius $a$ of the orbit $3.95 \times 10^{8} \mathrm{~km}$., the inclination of the orbit $51^{\circ}$, the magnitude 6.86 and the absolute magnitude $4 \cdot 0$, the effective temperature $6,570^{\circ} \mathrm{K}$. and the spectrum $F 5$. These figures refer to the primary component and are nearly the same for the secondary component, the mass of which is 0.959 that of the primary and its absolute magnitude $4 \cdot 1$. While the data indicate that the system is quite normal, the luminosities, according to the Bussell diagram, are lower than the average for the spectral type. 